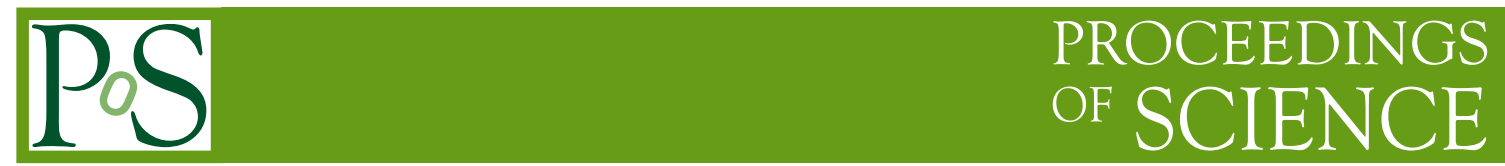

\title{
Recent results from ALICE
}

\author{
Yuri Kharlov*i \\ Institute for High Energy Physics, Protvino, 142281 Russian Federation \\ E-mail: Yuri.Kharlov@cern.ch
}

The ALICE experiment at the LHC has collected wealthy data in proton-proton and lead-lead collisions. An overview of recent ALICE results is given in this paper. Hadron spectra measured in pp collisions at $\sqrt{s}=0.9,2.76$ and $7 \mathrm{TeV}$ are discussed. Properties of hot nuclear matter produced in $\mathrm{Pb}-\mathrm{Pb}$ collisions at $\sqrt{s_{N N}}=2.76 \mathrm{TeV}$, revealed via many observables measured with the ALICE experiment, are shown.

LHC on the March,

November 16-18, 2011

Protvino, Moscow region, Russian Federation

\footnotetext{
${ }^{*}$ Speaker.

${ }^{\dagger}$ For the ALICE collaboration
} 


\section{Introduction}

The ALICE experiment was designed to study interactions of heavy ions at the LHC. This goal determines the unique performance of the ALICE detectors to reconstruct events with very high multiplicity and to measure spectra of identified hadrons, electrons, photons, muons in a wide energy range.

The data collected with the ALICE experiment with pp collisions at $\sqrt{s}=7 \mathrm{TeV}$ in 2010 2011 , consist of a minimum-bias sample with integrated luminosity $\int \mathscr{L} d T=16 \mathrm{nb}^{-1}$ and a sample recorded with rare-event triggers with integrated luminosity $\int \mathscr{L} d T=4.9 \mathrm{pb}^{-1}$. Rare-event triggers implemented pp collisions were based in EMCAL, PHOS and MUON detectors. Limited data samples with the proton beams at collision energies $\sqrt{s}=0.9$ and $2.76 \mathrm{TeV}$ have been also recorded with integrated luminosities $\int \mathscr{L} d T=0.14$ and $1.3 \mathrm{nb}^{-1}$ respectively.

The LHC has delivered heavy-ion collisions at the center-of-mass energy $\sqrt{s_{N N}}=2.76 \mathrm{TeV}$ to the ALICE experiment in 2010 with integrated luminosity $\int \mathscr{L} d T=10 \mu \mathrm{b}^{-1}$, and the data set of 2011 exceeded the previous one by an order of magnitude. Data taking of heavy ion collisions recorded in 2010 was dominated by the minimum bias trigger. In 2011, a fraction of minimum bias events was suppressed in favor of the triggers on the most central and semi-central events, as well as rare events which selected events with high-energy clusters in the electromagnetic calorimeters, muon tracks in the muon spectrometer, ultra-peripheral collisions.

\section{QCD tests in proton-proton collisions}

Properties of hot nuclear matter produced in heavy ion collisions are studied via a comprehensive set of observables. As a reference, similar observables are measured in proton-proton collisions. ALICE is performing detailed studies of hadron production spectra in pp collisions at all center-mass energies provided by the LHC. Apart of being a reference for heavy ion collisions, pp collisions is considered as a powerful tool for QCD studying. Advance particle identification capabilities [1] and a moderate magnetic field $(B=0.5 \mathrm{~T})$ allow to measure a variety of hadron spectra in a wide momentum range.

Identified charged hadron production in mid-rapidity are measured by the central tracking system consisting of the Inner Tracking System detector (ITS), Time Projection Chamber (TPC), Time-of-Flight detector (TOF) and a Cherenkov High-Momentum Particle Identification detector (HMPID). Each of these detectors provide particle identification in different complimentary momentum ranges, which allows to measure the spectra in a wide $p_{\mathrm{t}}$ range. ALICE has already published production spectra of $\pi^{ \pm}, K^{ \pm}, \mathrm{p}, \overline{\mathrm{p}}$ in pp collisions at $\sqrt{s}=0.9 \mathrm{TeV}$ [2], and has reported preliminary results on those spectra in pp collisions at $\sqrt{s}=7 \mathrm{TeV}$ [3]. Similar to charged hadrons, ALICE is able to measure neutral meson spectra by complimentary and redundant methods which ensures the result validity. Neutral pion and $\eta$-meson spectra were measured in pp collisions at all three LHC energies by the Photon Spectrometer (PHOS) which detected real photons and by the central tracking system which identifies photons converted to $e^{+} e^{-}$pairs on the medium of the inner ALICE detectors [4]. Combined analysis of all ALICE detectors allowed to measure spectra of resonance production, in particular strange and charmed hadrons. 
Results obtained by the ALICE on hadron production in pp collisions show a gradual increase of the mean transverse momentum with collision energy. Observed ratio between antiprotons and protons suggests that baryon-antibaryon asymmetry is restoring at high energies. Comparison of all measured spectra with Monte Carlo event generators and with next-to-leading perturbative QCD calculations demonstrates that no model can correctly describe spectra of hadron production at LHC energies.

\section{Global event properties in $\mathrm{Pb}-\mathrm{Pb}$ collisions}

Centrality of the collision, directly related to the impact parameter and to the number of nucleons $N_{\text {part }}$ participating in the collision, allows to study particle production versus density of the colliding system. Various ALICE detectors measure collision centrality, among them the best accuracy is achieved with the scintillator hodoscope VZERO covering pseudorapidity ranges $2.8<\eta<5.1$ and $-3.7<\eta<-1.7$. Distribution of the sum of amplitudes in VZERO in minimum bias $\mathrm{Pb}-\mathrm{Pb}$ collisions is shown in Fig.1 [5]. Centrality classes were defined by the Glauber model, and the fit of the Glauber model to the data is shown by a solid line in this plot.

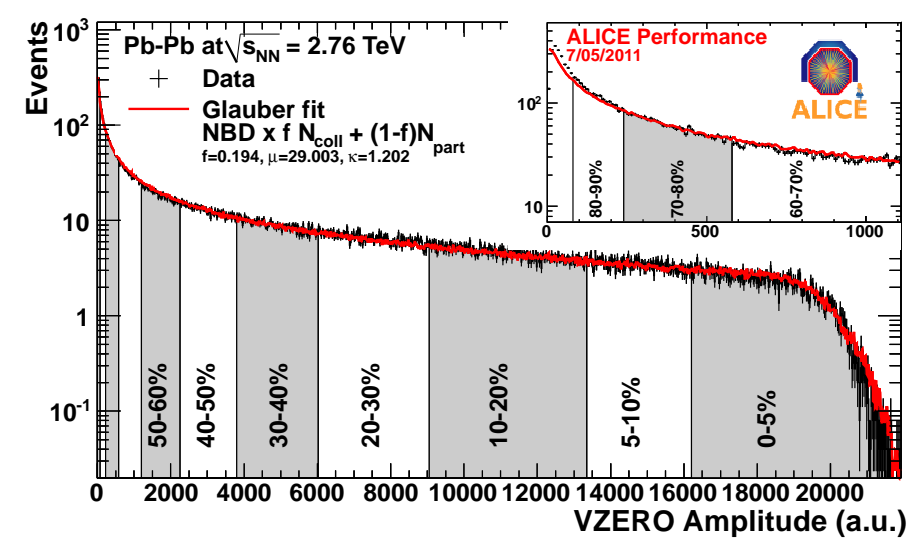

Figure 1: Centrality determination in ALICE. Glauber model fit to the VZERO amplitude with the inset of a zoom of the most peripheral region.

Charged particle multiplicity in $\mathrm{Pb}-\mathrm{Pb}$ collisions at $\sqrt{s_{N N}}=2.76 \mathrm{TeV}$ and its dependence on the collision centrality was measured with the Silicon Pixel Detector (SPD), two innermost layers of the barrel tracking system covering the pseudorapidity range $|\eta|<1.4$. The charged particle density, normalized to the average number of participants in a given centrality class, $d N_{\mathrm{ch}} / d \eta /\left(\left\langle N_{\text {part }}\right\rangle\right)$, measured by ALICE, was compared with similar measurements at lower energies at RHIC and SPS (Fig.2) [6]. In the most central events (centrality 0 - 5\%) at LHC energy the charged particle density was found to be $d N_{\mathrm{ch}} / d \eta=1601 \pm 60$ which is, being normalized to the number of participants, is 2.1 times larger than the charged particle density measured at RHIC at $\sqrt{s_{N N}}=200 \mathrm{GeV}$ and 1.9 times larger than that in pp collisions at $\sqrt{s}=2.36 \mathrm{TeV}$.

\section{Collective phenomena in heavy ion collisions}

The initial anisotropy of nuclei non-central collisions leads to anisotropic distribution on ini- 


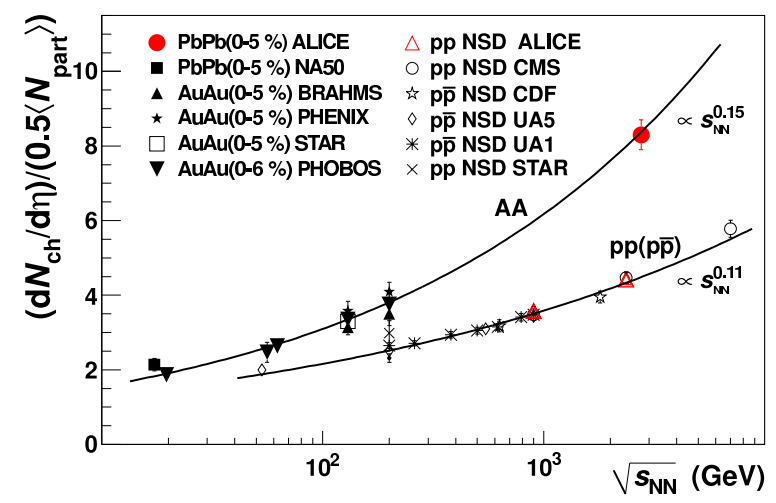

Figure 2: Charged track density $d N / d \eta$ in pp and AA collisions vs collision energy.

tial matter in the overlapping reagion. During evolution of the matter, the spatial asymmetry of initial state is converted to an anisotropic momentum distribution. The azimuthal distribution of the particle yield can be described by a Fourier series of a function of the angle between the particle direction $\varphi$ and the reaction place $\Psi_{\mathrm{RP}}$. The second coefficient of this series, $v_{2}$, is referred to as elliptic flow. Theoretical models, based on relativistic hydrodynamics [7, 8], successfully described the elliptic flow observed at RHIC and predict its increase at LHC energies from $10 \%$ to $30 \%$.

The first measurements of elliptic flow of charged particles in $\mathrm{Pb}-\mathrm{Pb}$ collisions at $\sqrt{s_{N N}}=$ $2.76 \mathrm{TeV}$ were reported by ALICE in [9]. Charged tracks were detected and reconstructed in the central barrel tracking system, consisting of ITS and TPC. Comparison of elliptic flow integrated over $p_{\mathrm{t}}$ measured by the ALICE and lower-energy experiments is shown in Fig.3. The observed

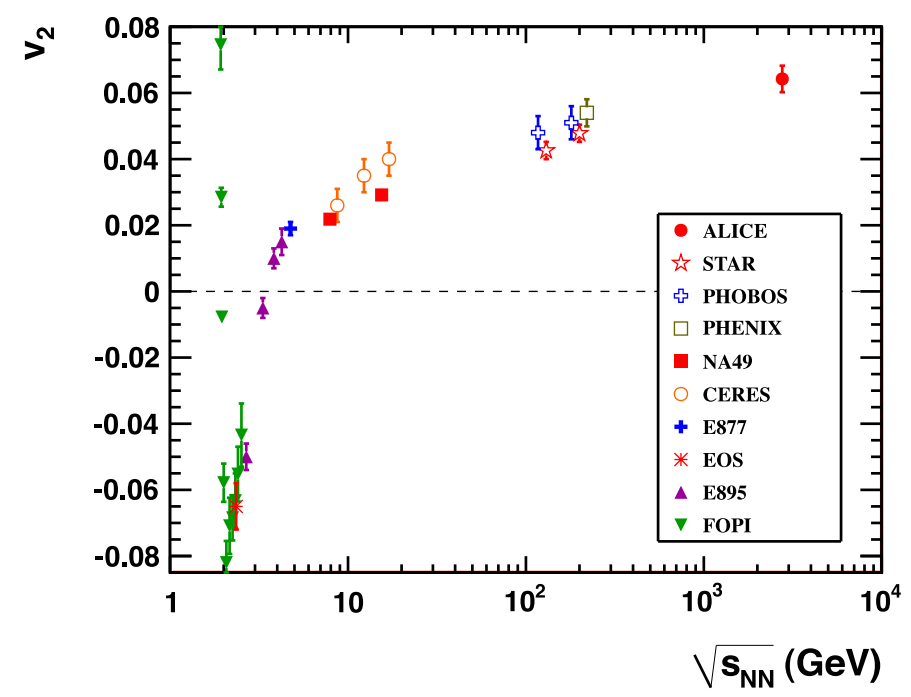

Figure 3: Azimuthal flow $v_{2}$ of charged particles measured by the ALICE in Pb-Pb collisions at $\sqrt{s_{N N}}=$ $2.76 \mathrm{TeV}$ in comparison with the lower-energy experiment results. 
trend of $v_{2}$ vs $\sqrt{s_{N N}}$ confirms model expectations that the value of $v_{2}$ in $\mathrm{Pb}-\mathrm{Pb}$ collisions at $\sqrt{s_{N N}}=$ $2.76 \mathrm{TeV}$ increases by about $30 \%$ with respect to $v_{2}$ in Au-Au collisions at $\sqrt{s_{N N}}=0.2 \mathrm{TeV}$.

The results of global event properties and collective expantion studied by ALICE, studied via the azimuthal anisotropy and intensity interferometry of identical particles [10], indicate that the fireball formed in nuclear collisions at the LHC is hotter, lives longer, and expands to a larger size at freeze-out as compared to lower energies.

\section{Strangeness production in heavy ion collisions}

Strange particle production has been considered as a probe of strongly interacting matter by heavy-ion experiments at AGS, SPS and RHIC. We have already demonstrated that ALICE, due to its powerful particle identification technique, has measured strange particle spectra in pp collisions. Similar analysis was performed on the $\mathrm{Pb}-\mathrm{Pb}$ data collected in 2010. Comparison of strange meson and baryon production is illustrated by the $\Lambda / K_{S}^{0}$ ratio measured by ALICE in different centrality classes (Fig.4, left). This ratio in peripheral $\mathrm{Pb}-\mathrm{Pb}$ collision is similar to that one measured in $\mathrm{pp}$ collisions, but it grows with centrality, increasing the value of 1.5 in the most central collisions. The qualitative behaviour of this ratio on $p_{\mathrm{t}}$ at the LHC collision energy is similar to the ratio measured at RHIC by the STAR experiment (Fig.4, right). An enhancement of strange and multi-strange
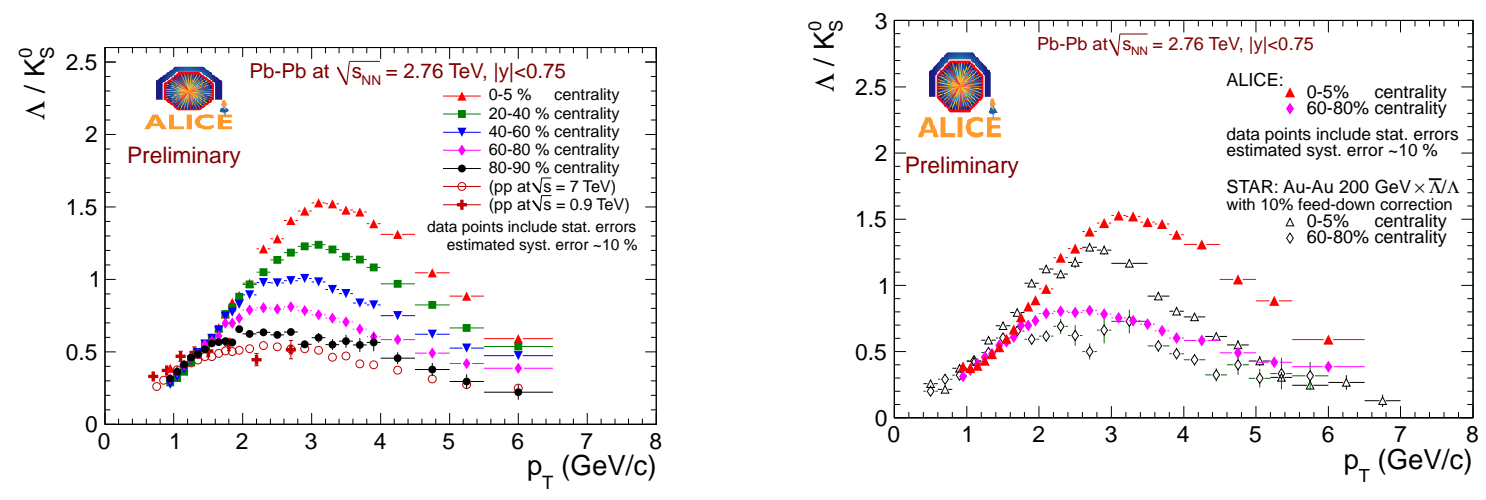

Figure 4: Ratio $\Lambda / K_{S}^{0}$ in $\mathrm{Pb}-\mathrm{Pb}$ collisions at $\sqrt{s_{N N}}=2.76 \mathrm{TeV}$ in different centralities (left) and comparison of this ratio at LHC and RHIC in centralities $0-5 \%$ and $60-80 \%$ (right).

baryons $\left(\Omega^{-}, \bar{\Omega}^{+}, \Sigma^{-}, \bar{\Sigma}^{+}\right)$was obsevred in heavy-ion collisions by experiments at lower energies, and was confirmed by ALICE at LHC energy [11]. It was also shown that multi-strange baryon enhancement scales with the number of participants $N_{\text {part }}$ and decreases with the collision energy.

The large yield of strange, and especially multi-strange baryons in heavy-ion collision was observed earlier at SPS and RHIC. This effect supports predictions of quark-gluon plasma formation which assumed that strange antiquarks are as abundant as light antiquarks in quark matter. The strange quark phase space becomes fully equilibrated, and therefore all strange hadrons are produced more abundantly. An overview of strangeness production in heavy ion collisions can be found in [12]. 


\section{Parton energy loss in medium}

Final-state partons produced at the initial stage of nucleus-nucleus collision, pass through medium with multiple secondary interactions. Energy loss by partons depends on density and temperature of the QCD medium. Hadrons produced in fragmentation of these partons should be suppressed compared to expectations from an independent superposition of nucleon-nucleon collisions. The strength of suppression of a hadron $h$ is expressed by the nuclear modification factor $R_{A A}$, defined as a ratio of the particle spectrum in heavy-ion collision to that in pp, scaled by the number of binary nucleon-nucleon collisions $N_{\text {coll }}$ :

$$
R_{A A}\left(p_{\mathrm{t}}\right)=\frac{\left(1 / N_{A A}\right) d^{2} N_{h}^{A A} / d p_{\mathrm{t}} d \eta}{N_{\mathrm{coll}}\left(1 / N_{p p}\right) d^{2} N_{h}^{p p} / d p_{\mathrm{t}} d \eta}
$$

Experiments at RHIC reported that hadron production at high transverse momentum in central Au$\mathrm{Au}$ collisions at a center-of-mass energy per nucleon pair $\sqrt{s_{N N}}=200 \mathrm{GeV}$ is suppressed by a factor $4-5$ with respect to pp collisions. At the larger LHC energy, the density of the medium is expected to be higher than at RHIC, leading to a larger energy loss of high- $p_{\mathrm{t}}$ partons. However, the hadron production spectra are less steeply falling with $p_{\mathrm{t}}$ at LHC than at RHIC which would reduce the value of $R_{A A}$ for a given value of the parton energy loss.

ALICE has measured the nuclear modification factor $R_{A A}$ for many particles. All charged particles, detected in the ALICE central tracking system (ITS and TPC), show a spectrum suppression [13] which is qualitatively similar to that observed at RHIC (Fig.5). However, quantitative comparison with RHIC demonstrates that the suppression at LHC energy is stronger which can be interpreted by a denser medium. Benefiting from particle identification which has been already mention earlier in this paper, ALICE has measured suppression of various identified hadrons, which provides experimental data for studying the flavor and mass dependence of the spectra suppression.

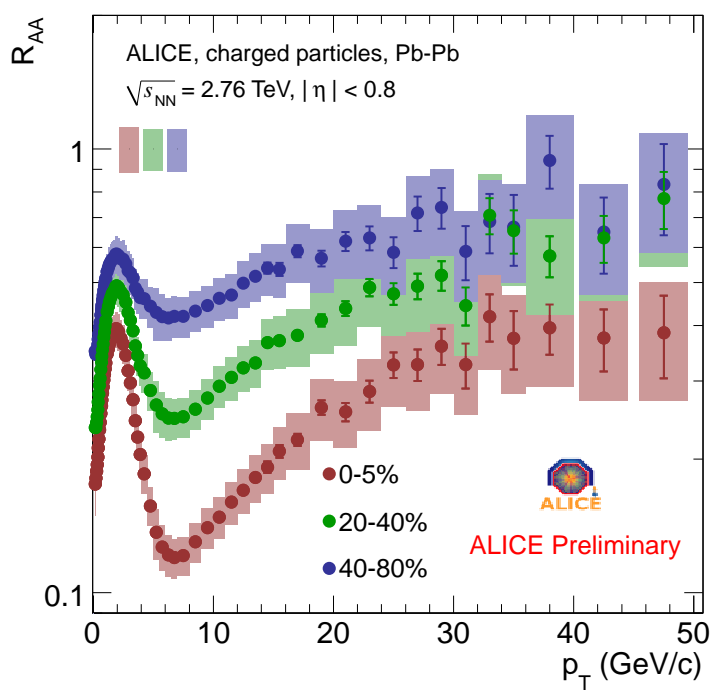

Figure 5: Nuclear modification factor $R_{A A}$ of charged particles. 


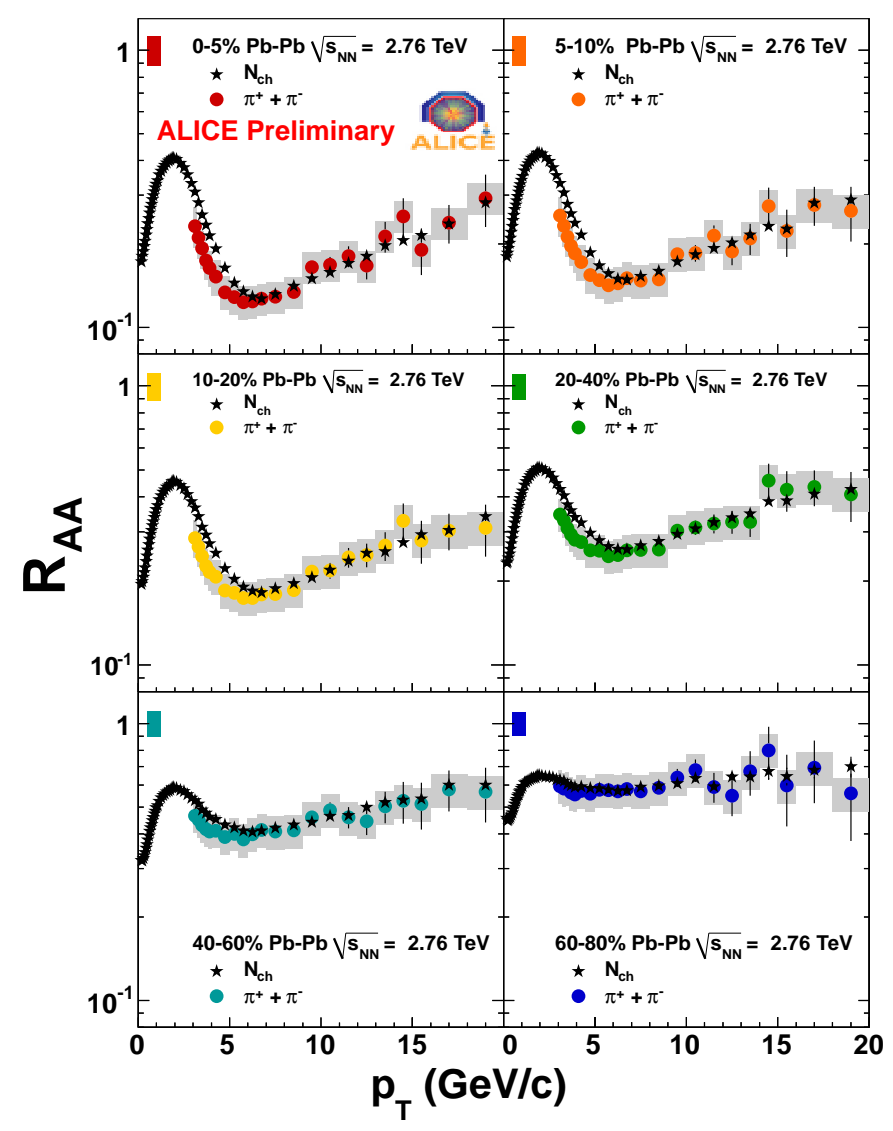

Figure 6: Nuclear modification factor $R_{A A}$ of charged pions.

A nuclear modification factor $R_{A A}$ of charged pion production in mid-rapidity (Fig.6) has lower values in the range of moderate transverse momenta $\left(3<p_{\mathrm{t}}<7-10 \mathrm{GeV} / c\right)$ than that of unidentified charged particles, but at higher $p_{\mathrm{t}}$ it coincides with all charged particles. To the contrary to charged pions, strange hadrons $\left(K_{S}^{0}, \Lambda\right)$ are less suppressed in the most central collisions compared to all charged particles (Fig.7). This is explained by the fact that strange quark production is enhanced in a hot nuclear medium, and this strangeness enhancement partially compensates energy loss of strange quarks, such that the overall $R_{A A}$ value becomes larger than for pions. Lambda hyperons have no suppression at $p_{\mathrm{t}}<3-4 \mathrm{GeV} / c$, which is interpreted by an additional baryon enhancement in central heavy-ion collisions.

ALICE has reported also the first measurements of $D$ meson suppression [14] in $\mathrm{Pb}-\mathrm{Pb}$ collisions in two centrality classes, $0-20 \%$ and $40-80 \%$, shown in Fig.7. It was shown that the $R_{A A}$ values for $D^{0}, D^{+}$and $D^{*+}$ are consistent with each other within the statistical and systematical uncertainties. Although the statistics of the ALICE run 2010 is marginal for $D$ meson measurement, the obtained result shows a hint that the $D$ mesons are less suppressed than charged pions.

Nuclear modification factor $R_{A A}$ is $J / \psi$ production in $\mathrm{Pb}-\mathrm{Pb}$ collisions was measured by the ALICE in two kinematic regions: in the forward rapidity with the muon spectrometer and in mid- 

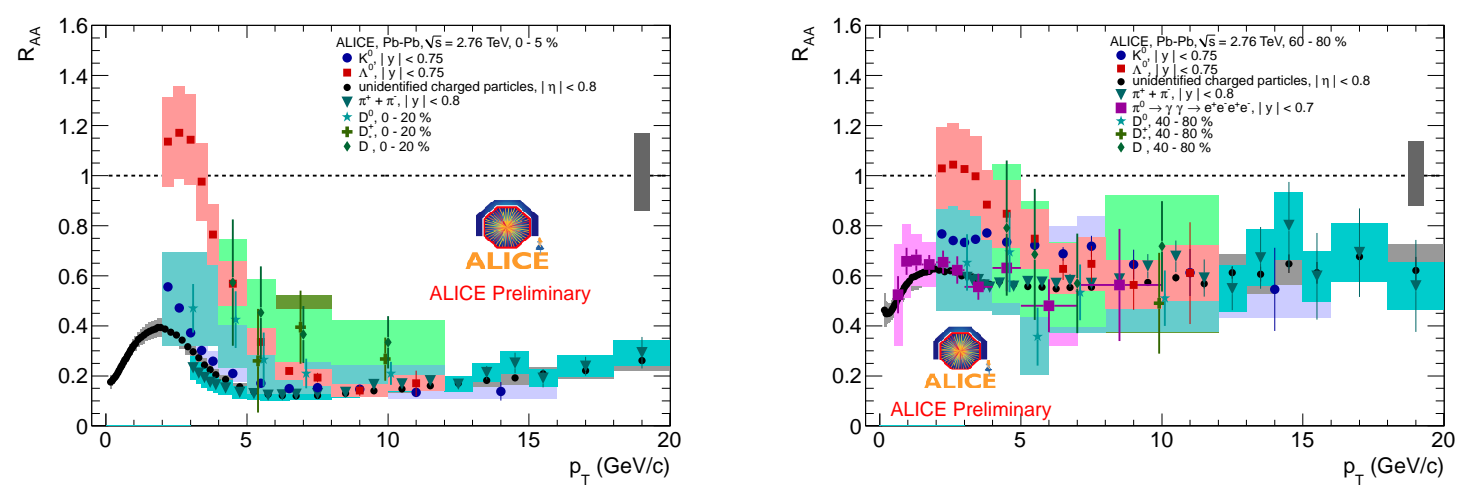

Figure 7: Nuclear modification factor $R_{A A}$ of charged particles, $K^{0}, \Lambda, \pi^{ \pm}, D^{+}, D^{0}, D^{*+}$ in central (left) and peripheral (right) collisions.

rapidity deploying the central tracking system [15]. The result of $R_{A A}$ measurement in forward rapidity shows almost no dependence on collision centrality with the average value $R_{A A}=0.545 \pm 0.032$ (stat.) \pm 0.084 (syst.) which is significantly different from the RHIC results.

\section{Conclusion}

The ALICE collaboration is performing QCD studies via hadron production measurements in proton-proton collisions. Obtained results in pp collisions at $\sqrt{s}=0.9,2.76$ and $7 \mathrm{TeV}$ show statistically significant deviations from models which well described lower-energy results. Therefore new experimental results from pp collision allow to tune various phenomenological models and pQCD calculations.

Comprehensive studies of heavy-ion collisions, performed by the ALICE experiment show that the properties of strongly interacting nuclear matter produced at the LHC energy, qualitatively similar to those observed at RHIC and reveal smooth evolution with collision energy. The matter produced at LHC has about 3 times larger energy density, twice larger volume of homogeneity and about 20\% larger lifetime. Like at RHIC, the matter at LHC reveals the properties on an almost perfect liquid. Particle suppression appeared to be stronger at LHC than at RHIC which is also an evidence of denser medium produced at LHC.

\section{References}

[1] ALICE Collab. (K. Aamodt et al.), The ALICE experiment at the CERN LHC, JINST 3, S08002 (2008).

[2] ALICE Collab. (K. Aamodt et al.), Eur. Phys. J. C 71(6), 1655 (2011).

[3] R. Preghenella for the ALICE Collab., Identified-particle production and spectra with the ALICE detector in pp and Pb-Pb collisions at the LHC,arXiv:1111.7080v1 [hep-ex]; B. Guerzoni, PoS(IHEP-LHC-2011)003.

[4] ALICE Collab., Neutral pion and $\eta$ meson production in proton-proton collisions at $\sqrt{s}=0.9 \mathrm{TeV}$ and $\sqrt{s}=7 \mathrm{TeV}$, CERN-PH-EP-2012-001, CERN, Geneva, 2012. 
[5] ALICE Collab. (K. Aamodt et al.), Phys. Rev. Lett. 106, 032301 (2011).

[6] ALICE Collab. (K. Aamodt et al.), Phys. Rev. Lett. 105, 252301 (2010).

[7] G. Kestin and U. W. Heinz, Eur. Phys. J. C 61, 545 (2009).

[8] H. Niemi, K. J. Eskola and P. V. Ruuskanen, Phys. Rev. C 79, 024903 (2009).

[9] ALICE Collab. (K. Aamodt et al.), Phys. Rev. Lett. 105, 252302 (2010).

[10] ALICE Collab. (K. Aamodt et al.), Phys. Lett. B 696, 328 (2011).

[11] B. Hippolyte for the ALICE Collab., Strange and multi-strange particle production at the LHC energies with ALICE, arXiv:1112.5803 [nucl-ex].

[12] B. Muller, Strangeness and the Quark-Gluon Plasma: Thirty Years of Discovery, arXiv:1112.5382v1 [nucl-th].

[13] J. Otwinowski for the ALICE Collab., Charged Particle Production at Large Transverse Momentum in Pb-Pb Collisions at $\sqrt{s_{N N}}=2.76$ TeV Measured with ALICE at the LHC, J. Phys. G 38, 124112 (2011); arXiv:1110.2985 [hep-ex].

[14] A. Grelli for the ALICE Collab., Heavy flavour physics with the ALICE detector at the CERN-LHC, J. Phys. Conf. Ser. 316, 012025 (2011). R. Averbeck for the ALICE Collab., PoS(IHEP-LHC-2011)014; arXiv:1202.6329v1 [nucl-ex].

[15] F. Bossu for the ALICE Collab., PoS(IHEP-LHC-2011)015. 